\title{
Survey on Comparative Analysis of Various Image Compression Algorithms with Singular Value Decomposition
}

\author{
Poonam Dhumal \\ Department of Computer \\ Engineering,Pimpri-Chinchwad \\ College of Engineering, \\ Pune-411044
}

\author{
S. S. Deshmukh \\ Department of Computer Engineering, \\ Pimpri-Chinchwad College of Engineering, \\ Pune-411044
}

\begin{abstract}
Image compression techniques are the most apprehensive topics in today's high-tech environment. Singular Value Decomposition (SVD) is one of the image compression technique. SVD is an attractive algebraic transform for digital image processing applications. The SVD method can transform matrix A into product, which allows us to refactor a digital image in three orthogonal matrices. The using of singular values of such refactoring allows us to represent the image with a reduced set of values, which can store the useful features of the given original image, also use less storage space of the memory, and achieve the image compression process. In this paper, discuss how SVD is applied to images, the technique of image compression and maintain the quality of the image using SVD and also the algorithm to compress an image using MATLAB.
\end{abstract}

\section{Keywords}

Image processing Singular Value Decomposition (SVD); Image compression; MATLAB;

\section{INTRODUCTION}

Now a day, everyone is fond of collecting the photos, images and videos. Not only photos, man wish to capture all his unforgettable moments. This results in the increase of number of images and videos. It is obvious that a more amount of memory and quality of image is needed to store all these images and videos. If these images are needed to be transmitted, it also requires large bandwidth and quality. So, for that it need of image compression techniques. These image compression techniques reduce the disk storage space occupied by the image without any loss to image quality [4]

Thus the image size can be reduced by selecting proper image compression technique liable on the requirement of the user or application. Many of the image processing techniques were developed with application to medical imaging, object recognition, face recognition, satellite Imagery, and photo enhancement [4].

\subsection{Image Compression}

Digital image compression technique which compares the image which reduce disk space with maintaining the quality of the image. Basically an image compression technique is divided into two classes:

\subsubsection{Lossy Image Compression}

In lossy image compression, image is compressed with having some loss of data. After applying decompression original image cannot reconstruct. SVD is the lossy image compression technique.

\subsubsection{Lossless image compression}

In lossless image compression it generates the compressed image without loss. Which is same as the original image.

\subsection{Singular Value Decomposition Thechnique}

In image processing domain Singular Value Decomposition (SVD) is said to be a significant topic in linear algebra by many famous mathematicians. SVD has many practical and theoretical values; Special features of SVD are that it can be performed on any input $(\mathrm{m}, \mathrm{n})$ matrix. We have an input matrix $A$ with $m$ rows and $n$ columns, with rank $r$ with $\mathrm{r} \leq n \leq$ $m$. Then the input matrix A can be factored into three diagonal matrices: $A=S V D^{T}$

Every image is represented by a number of pixel values. Pixels represent the intensity of the given image. These pixel values are arranged as a matrix form with rows and columns. The matrix representation of an image can be easily obtained using MATLAB

The key to working with SVD of any given matrix $\mathrm{A}$ is to consider $A A^{\mathrm{T}}$ and $A^{\mathrm{T}} A$. The columns of $\mathrm{U}$, that is $\mathrm{m}$ by $\mathrm{m}$, are eigenvectors of $\mathrm{AA}^{\mathrm{T}}$, The columns of $\mathrm{V}$, that is $\mathrm{n}$ by $\mathrm{n}$, are eigenvectors of $\mathrm{A}^{\mathrm{T}} \mathrm{A}$. The singular values on the diagonal of matrix $\mathrm{S}$, that is $\mathrm{m}$ by $\mathrm{n}$, are the positive square roots of the nonzero eigenvalues of both $\mathrm{AA}^{\mathrm{T}}$ and $\mathrm{A}^{\mathrm{T}} \mathrm{A}$.

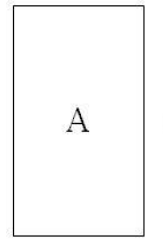

$m \times n$

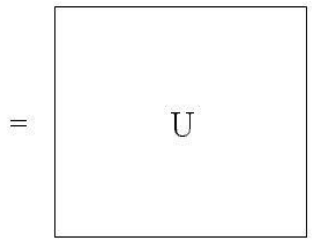

$m \times m$

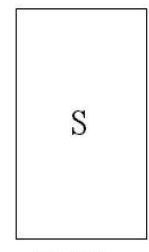

$m \times n$

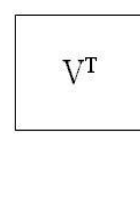

$n \times n$
Where $\mathrm{A}$ is the image matrix $\mathrm{A}_{m \times n}$;

Where $\mathrm{A}$ is the image matrix $\mathrm{A}_{m \times n}$;

Where Matrix $U$ is a $m \times m$ orthogonal matrix

$\mathrm{U}=\left[\begin{array}{lllllll}u_{1} & u_{2} & \ldots & u_{r} & u_{r+1} & \ldots . & u_{m}\end{array}\right]$ 
Here, $S$ is a $m \times n$ diagonal matrix which is not orthogonal matrix with singular values (SV) on the diagonal. The matrix $S$ can be shown in following

$$
S=\left[\begin{array}{ccccccc}
\sigma_{1} & 0 & \cdots & 0 & 0 & \cdots & 0 \\
0 & \sigma_{2} & \cdots & 0 & 0 & \cdots & 0 \\
\vdots & \vdots & \ddots & \vdots & \vdots & \ddots & \vdots \\
0 & 0 & \cdots & \sigma_{r} & 0 & \cdots & 0 \\
0 & 0 & \cdots & 0 & \sigma_{r+1} & \cdots & 0 \\
\vdots & \vdots & \ddots & \vdots & \vdots & \ddots & \vdots \\
0 & 0 & \cdots & 0 & 0 & \cdots & \sigma_{n} \\
0 & 0 & \cdots & 0 & 0 & \cdots & 0
\end{array}\right]
$$

For $i=1,2, \ldots, n, \boldsymbol{i} \mathrm{s}$ are called Singular Values

(SV) of the matrix $A$.

And matrix $V$ is an $n \times n$ orthogonal matrix

$\mathrm{V}=\left[\begin{array}{lllllll}v_{1} & v_{2} & \ldots & v_{r+1} & v_{r} & \ldots \ldots & v_{n}\end{array}\right]$

column vectors $i \mathbf{v}$ for $\mathrm{i}=1,2, \ldots, n$, form an

Orthogonal sets.

1. The matrices $U$ and $V$ are not Unique, however, The singular value $\sigma_{1,}, \sigma_{2}, \ldots \sigma_{n}$ are unique.

2. Since $\mathrm{A}^{T} \mathrm{~A}=\mathrm{VS}^{T} \mathrm{SV}^{T}$, so $\mathrm{V}$ diagonalize $\mathrm{A}^{T} \mathrm{~A}$ , it follows that the $\mathrm{v}^{j} \mathrm{~s}^{\prime}$ are the eigenvector of $\mathrm{A}^{T}$ A .

\section{RELATED WORK}

In literature, Ozcelik and A. K. Katsaggelos [1] proposed a mean field annealing method for reducing objects. To reduce artifacts while keeping the required detail present in the primeval image. Proposed technique makes use of a priori information about the pristine image through a no stationary Gauss-Markov model. A maximum a posteriori (MAP) estimation is getting iteratively utilizing mean field annealing [1].

Bredies and Holler [3] proposed a total variation decompression model for reducing artifacts. A fast primal dual algorithm is developed to solve this model effectively; It is one of the energy minimization methods.. The work [4], [5] the K-SVD method developed to reduce the artifacts present in the image after decompression by improving PSNR. But the existing methodology (K-SVD algorithm) is quite computationally demanding, especially when the dimensions of the dictionary rise or the number of training signals converts big [3].

Rowayda A. Sadek [5] proposed contribution in using unused SVD characteristics in novel approaches such as an adaptive block based compression, perceptual multiple watermarking, image capacity for thumping information, irregularity measure, etc., All these contributions were experimentally examined and gave talented results compared to established ones. The main contributions are a novel perceptual image forensic technique, a new potential visualization in using the SVD Properties, reviewing and experimental valuation of the developed SVD based application such as compression, a new block based roughness measure for application such as perceptual liberal compression as well as perceptual progressive data hiding [5].

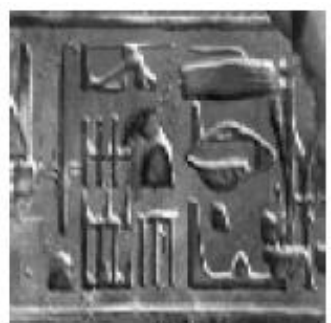

(a)

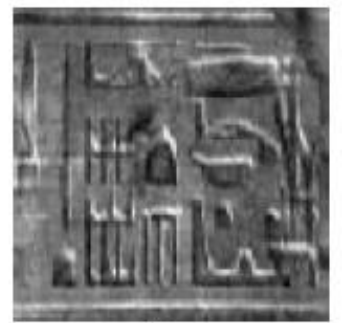

(b)
Figure 2. Truncated SVD a) original image b) truncated SVD

Neethu. K. J [6] proposed Improved Quality of JPEG Compressed Image Using Approximate K-SVD Algorithm. JPEG compressed images contain ringing and blocking artifacts, which can be offensive to the viewer above certain compression ratios. The quality of compressed image can be evaluated qualitatively and quantitatively. PSNR and SSIM are usually used by quantitative evaluation of image quality [6].

a) PSNR: Peak Signal to Noise Ratio (PSNR) is defined as a relation among the full probable power of a signal and the power of corrupting noise that affects the fidelity of its representation. In image compression Signal is the pristine data or image, and noise is the fault presented by compression of image. It is stated in terms of logarithmic decibel measure.

b) SSIM: The Structural Similarity index (SSIM) is a method for checking the similarity between two images. SSIM considers image degradation as perceived changes in structure Detail.

Table 1. Related work table

\begin{tabular}{|c|c|c|c|}
\hline Method & PSNR & SSIM & $\begin{array}{c}\text { Computatio } \\
\text { nal Time }\end{array}$ \\
\hline $\begin{array}{c}\text { Mean Field } \\
\text { Annealing } \\
\text { image [1] }\end{array}$ & High & Average & Average \\
\hline $\begin{array}{c}\text { Post } \\
\text { processing on } \\
\text { image [2] }\end{array}$ & High & Average & Average \\
\hline $\begin{array}{c}\text { Total } \\
\text { variation } \\
\text { Regularizatio } \\
\text { n between } \\
\text { image [3] }\end{array}$ & High & Average & Average \\
\hline $\begin{array}{c}\text { Classical K- } \\
\text { SVD result } \\
\text { [4] }\end{array}$ & High & Average & Average \\
\hline $\begin{array}{c}\text { KSVD+TV } \\
\text { [5] }\end{array}$ & High & High & High \\
\hline
\end{tabular}

\subsection{Algorithm}

SVD algorithm for image Compression

Step-1

Read the image (input image).

Step-2

Convert the input image (color image) into a gray scale image which is reduce in size. 


\section{Step-3}

Decompose each component using Singular Value Decomposition i.e. SVD.

\section{Step-4}

Select $k$ value and discard the diagonal value of $\mathrm{S}$ matrix that are not required. And Construct the image using the selected singular values.

The $\mathrm{k}$-value in the $\mathrm{m}$-file represents the number of iterations taken on each layer used in the resulting disintegration. This Is really the rank of the SVD matrix. By increasing the rank we can increase clarity till an ideal image is got.

\section{Step-5}

Show the compressed image.

\section{RESULT}

After applying various ' $k$ ' singular values, generates the different image compression result.

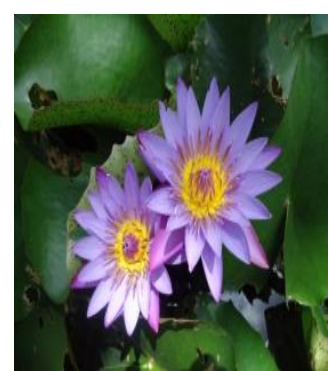

a)Original Image

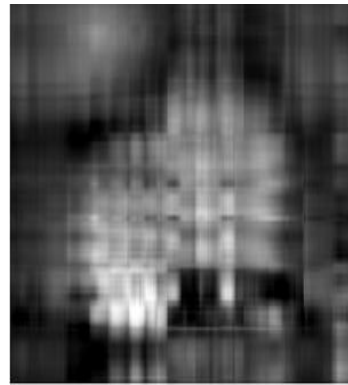

c)Singular Value $k=5$

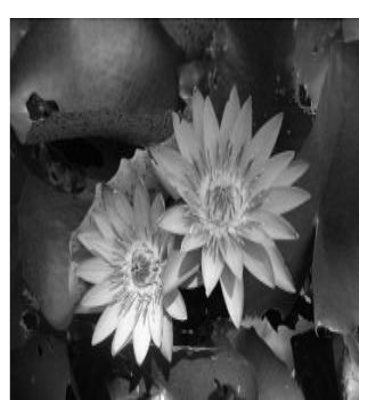

e) Singular Value k=155

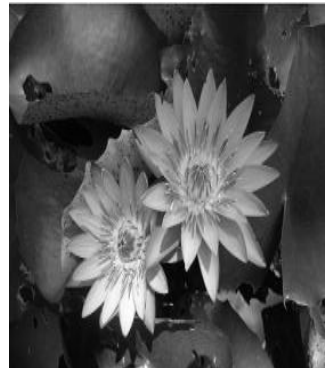

b)Gray Scale image

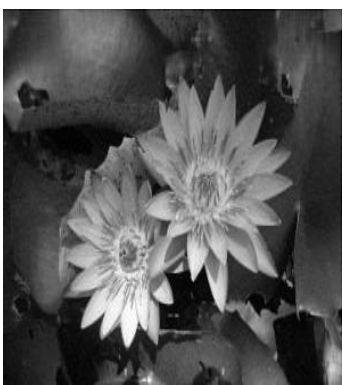

d) Singular Value $k=80$

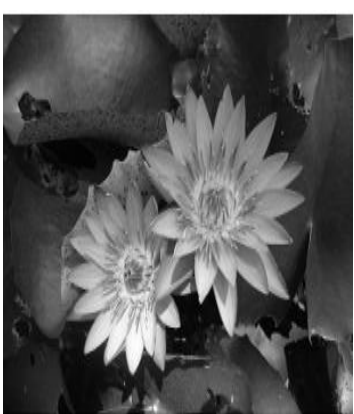

f) Singular Value $k=280$

\subsection{Experimental Evaluation}

Following a graph shows comparison of error between compressed and original image with different singular values

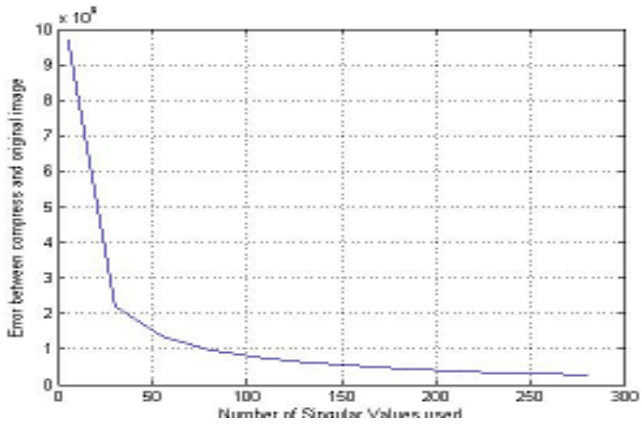

4. CONCLUSION

This paper is for a practical survey of SVD characteristics in various developed image processing approaches. Survey in using unused SVD characteristics in new approaches such as an adaptive block based compression, these contributions were experimentally checked and gave promising results compared to developed SVD. Singular Value Decomposition (SVD) is a very simple, robust and reliable technique. This SVD image compression technique provides a stable and effective method to divide the image matrix into a set of linearly independent matrices to get the different singular values.

The basis of the theory and results of experiments, we found that SVD is a constant and effective method to divide the system into a set of linearly independent components, each of them is resounding own information to give to the system, Thus, both ranks of the problem and subspace orientation can be determined. The JPEG image compression scheme is a widely used form of compression technique which provides better image compressed result. SVD provides good compression ratio and also a practical solution to image compression problem from PSNR, SSIM ratio. The results shown above clearly display the compressed output for different $\mathrm{k}$ values. Thus, selection of $\mathrm{k}$ value plays an important role in this SVD based image compression technique. Finally, get the compressed image with better quality.

\section{REFERENCES}

[1] T. Ozcelik, J. Brailean, and A. Katsaggelos, Image and video compression algorithms based on recovery techniques using mean field annealing," Proceedings of the IEEE, vol. 83, no. 2, pp. 304-316, 1995.

[2] M.-Y. Shen and C.-C. J. Kuo, Review of postprocessing techniques for compression artifact removal," Journal of Visual Communication and Image Representation, vol. 9, no. 1, pp. 2-14, 1998.

[3] K. Bredies and M. Holler, Artifact-free jpeg decompression with total generalized variation." in VISAPP (1), pp. 12-21, 2012.

[4] K. Mounika, D. Sri Navya Lakshmi, K. Alekya, SVD based image compression," International Journal of Engineering Research and General Science Volume 3, Issue 2, March-April,2015"

[5] Rowayda A. Sadek, SVD Based Image Processing Applications: State of The Art, Contributions and Research Challenges," (IJACSA) International Journal of Advanced Computer Science and Applications, Vol. 3, No. 7, 2012" [6] Guoliang Zeng, "Face Recognition with Singular Value Decomposition.”, CISSE Proceeding, 2006 
[6] Neethu.K.J, Sherin Jabbar,Improved Quality of JPEG Compressed Image," IEEE Sponsored 2nd International Conference on Innovations in Information Embedded and Communication Systems ICIIECS'15" Using Approximate K-SVD Algorithm

[7] Rafael C. Gonzalez, Richard E. Woods, Steven L. Eddins, "Digital Image Processing Using MatLab", Prentice Hall, 2006
[8] Bernd Jahne, "Digital Image Procession", Springer, 2002

[9] Steve J. Leon; "Linear Algebra with Applications", Macmillan Publishing Company, New York; 1996

[10] Lijie Cao," Singular Value Decomposition Applied To Digital Image Processing", 